\title{
Effects of musical training and event probabilities on encoding of complex tone patterns
}

\author{
Anja Kuchenbuch', Evangelos Paraskevopoulos ${ }^{1}$, Sibylle C Herholz ${ }^{2,3}$ and Christo Pantev
}

\begin{abstract}
Background: The human auditory cortex automatically encodes acoustic input from the environment and differentiates regular sound patterns from deviant ones in order to identify important, irregular events. The Mismatch Negativity (MMN) response is a neuronal marker for the detection of sounds that are unexpected, based on the encoded regularities. It is also elicited by violations of more complex regularities and musical expertise has been shown to have an effect on the processing of complex regularities. Using magnetoencephalography (MEG), we investigated the MMN response to salient or less salient deviants by varying the standard probability (70\%, 50\% and 35\%) of a pattern oddball paradigm. To study the effects of musical expertise in the encoding of the patterns, we compared the responses of a group of non-musicians to those of musicians.

Results: We observed significant MMN in all conditions, including the least salient condition (35\% standards), in response to violations of the predominant tone pattern for both groups. The amplitude of MMN from the right hemisphere was influenced by the standard probability. This effect was modulated by long-term musical training: standard probability changes influenced MMN amplitude in the group of non-musicians only.

Conclusion: This study indicates that pattern violations are detected automatically, even if they are of very low salience, both in non-musicians and musicians, with salience having a stronger impact on processing in the right hemisphere of non-musicians. Long-term musical training influences this encoding, in that non-musicians benefit to a greater extent from a good signal-to-noise ratio (i.e. high probability of the standard pattern), while musicians are less dependent on the salience of an acoustic environment.
\end{abstract}

Keywords: Processing of tone patterns, Mismatch negativity, Musical training, MEG

\section{Background}

The capacity for detecting unexpected and possibly important or dangerous events is essential for adaptive behaviour. The human auditory cortex automatically encodes acoustic input from the environment and differentiates regular sound patterns from deviant ones in order to identify possibly important, irregular events, such as a sudden change in the sound of a car that might indicate engine malfunction.

The Mismatch Negativity (MMN) response is a marker for the detection of sounds that are unexpected given a basis of previously encoded regularities. It has been widely used to investigate the processing of auditory stimuli in the auditory cortex in healthy and clinical

\footnotetext{
*Correspondence: pantev@uni-muenster.de

'Institute for Biomagnetism and Biosignalanalysis, University of Münster, Münster, Germany

Full list of author information is available at the end of the article
}

populations [1-5]. MMN-like responses have also been reported in animals [6-8], which have further elucidated the physiological basis of the MMN response.

The MMN is elicited not only by violations of simple acoustic features or local-features, such as pitch or timbre, but also by violations of more complex or global regularities. To detect violations of local regularities, a current auditory event has to be compared constantly to an established regularity. The comparator mechanism underlying the MMN has therefore been described as a memory-based process and the temporal window of integration (TWI) of echoic memory is typically assumed to be around 10s [9]. There is evidence that global regularities, rather than local regularities, can also determine whether a tone is perceived as a violation and can therefore elicit an MMN response [10-13]. For example, a pattern regularity like "W" is followed by tone "Y" is followed by tone "Z" is not based on the repetition of 
one repeatedly presented tone but instead on the sequence of 3 different tones which have to be grouped in order to establish the pattern regularity. If the pattern is more complex and is additionally presented at a rather low frequency, the establishment of the pattern regularity exceeds the TWI and cannot be based on local regularities, but rather rely on higher-order memory systems that can integrate over a longer time frame than 10 s. Herholz and colleagues, for example, found that expec tancies of tones in a continuous tone stream are established by global regularities (tone patterns) and that the MMN elicited in response to violations of the established regularity is based on global statistical knowledge rather than a local memory span. In conclusion, the MMN represents a more general violation detection mechanism that does not necessarily need to be based on local regularities. The elicited MMN could be found in response to violations of the predominant standard pattern occurring with a probability of only 50\% [12].

Evidence from an animal study supports this idea: a single-neuron phenomenon, a decrease in the response to a repeated stimulus, which does not generalize to other stimuli, called stimulus-specific adaption (SSA) has been associated with MMN. Studying neurons in the primary auditory cortex of cats, Ulanovsky and colleagues (2004) found that responses to tone-pattern-like sequences of tones $\mathrm{A}$ and $\mathrm{B}$ (for example BBA, ABA) depend upon the overall stimulus probability: by decreasing deviant probability (10\%-90\% deviant probability), and therefore increasing standard probability, the neuronal response increases [14]. Other studies on SSA in rats and rodents show that SSA is a different process than the human MMN, since neurons do not generate a late deviant response component directly equivalent to the human MMN [15,16]. Recent investigations by Sculthorpe and Campbell (2011) into the way in which the MMN response in tone patterns is influenced by different rare deviant probabilities $(0.02$ to $0.16 \%$ deviant probability) of the violating event, and thereby different probabilities of the standard event, concluded that the MMN amplitude does not vary with deviant probability [17] under these conditions. All deviant probabilities were, however, very low (and the standard probabilities, accordingly, very high) compared to the wider range of event probabilities used in the experiment of Ulanovsky et al. (2004). Various studies show that simple feature MMN is influenced by standard probability, through an inverse relationship between MMN amplitude and deviant probability [18-22]. The lower threshold of standard probability that is required for the successful encoding within the auditory cortex of an auditory pattern (standard probability) among other tones in an acoustically variable environment is, however, unknown.
Expertise shapes brain anatomy and brain physiology. Therefore experts often show greater abilities within their field of expertise than do non-experts. Navigation abilities, for example, have been associated with relative increase in posterior hippocampus grey matter volume accompanied by relative decrease in anterior hippocampus in London taxi drivers. Expert chess players have been found to activate different brain systems than novices [23-27]. In a longitudinal study, Hyde and colleagues found structural brain changes after the relatively short time span of only 15 months of musical training, which were correlated with improvements in musically-relevant motor and auditory skills [28]. By enabling the comparison of experts and non-experts within the auditory domain (musicians versus non-musicians, respectively), musical training has been recognized as an important tool for the investigation of long term training-driven plasticity effects and enhanced auditory processing [29-39]. The processing of sound patterns has also been shown to be improved by long-term musical training $[12,13,36,40,41]$ and tends to be leftlateralized $[12,13,40]$. It is, however, still unknown how musical experience affects the encoding of patterns that are difficult to extract from an acoustically variable environment.

In the current experiment, we used magnetoencephalography (MEG) to investigate the effect of long-term musical training on the processing of tone patterns that varied in salience within their acoustic context. Note: The term salience or saliency is used throughout this study in its original meaning, that is, the state or condition of being prominent or most noticeable or important, and not in its special meaning in perceptual psychology (i.e. the state or quality by which it stands out relative to its neighbors). We compared musicians' and non-musicians' auditory processing in three conditions which differed in the frequency of the standard pattern: salient (70\% probability of the standard pattern occurring), less salient (50\% probability of the standard pattern) and least salient (35\% probability of the standard pattern). In each of these conditions, four deviant patterns with approximately equal probability were presented. The probability of any individual deviant (i.e. deviant pattern) occurring was lower than that of the standard pattern. The deviant and standard patterns were presented randomized as a continuous tone stream. This allowed us to test the following: at which probabilities of regular tone pattern ("standards") could MMN responses to violations of this pattern ("deviants") be observed at all (i.e. the lower threshold of standard probability); how the MMN response is affected by standard probability in tone patterns; and how musical expertise affects the encoding of the tone patterns. Additionally, a standard frequency oddball condition was 
recorded in which no differences between the groups were expected.

\section{Results}

\section{MEG data}

Amplitudes and latencies of the MMN were entered into statistical analyses. In all analyses the alpha level was 0.05 and tests were two-tailed.

\section{Effect of standard probability and hemisphere on the MMN}

To test in which experimental conditions a significant MMN deflection to the pattern deviants was elicited, we performed nonparametric bootstrapping tests (1000 resampling iterations) on the group averaged waveforms for the MMN in both hemispheres for all three pattern conditions [42-45]. Time windows in which the 0.95 percent confidence interval of the bootstrap did not include zero values were considered to indicate significant deflections. As shown in Figure 1, the MMN deflection is significantly different from zero in all three pattern conditions in both groups.

The amplitudes of the individual MMN difference source waveform peaks of the three pattern condition (pattern deviant) were entered into a mixed model $2 \times$ $2 \times 3$ ANOVA with the between subject factor being group (musicians and non-musicians) and the within subject factors being hemisphere (left and right) and standard probability (35\%, 50\% and 70\%).

For the MMN amplitude we found a significant main effect of the standard probability $[F(2,68)=3.817$, $\mathrm{p}=.027]$, and a 2-way-interaction of hemisphere $\times$ standard probability $[\mathrm{F}(2,68)=4.742, \mathrm{p}=.012]$ as well as a 3 -way-interaction of hemisphere $\times$ standard probability $\times$ group $[\mathrm{F}(2,68)=5.331, \mathrm{p}=.007]$. In order to explore the source of significant interaction we conducted additional ANOVAs: amplitude was entered into repeatedmeasures $2 \times 3$ ANOVAs using within subject factors of hemisphere (left and right) and standard probability (35\%, 50\% and 70\%) for each group (musicians and nonmusicians). The results showed no significant main effects or interactions within the group of musicians, while the results within the group of non-musicians revealed differences a main effect of standard probability [F $(2,34)=5.050, \mathrm{p}=.012])$ as well as difference in lateralization of the MMN for specific standard probability (2-way-interaction of hemisphere $\times$ standard probability $[\mathrm{F}(2,34)=9.550, \mathrm{p}=.001])$. To understand the effect of the 3-level factor standard probability we conducted 2 repeated-measures 1-way-ANOVAs for the group of non-musicians for each hemisphere (left and right) with the factor standard probability $(70 \%, 50 \%$ and $35 \%$ standard probability, respectively) and to analyze the effect of hemisphere on MMN amplitude separately for each standard probability, afterwards paired-samples t-tests for non-musicians were conducted to compare the two hemispheres (left and right) in amplitude for every standard probability (70\%, 50\% and 35\% standard probability, respectively). Because both analyses are using the same data the alpha level was divided by two $(\mathrm{p}<.025)$ in order to correct for multiple comparison. The results of the 1-way-ANOVAs show a significant

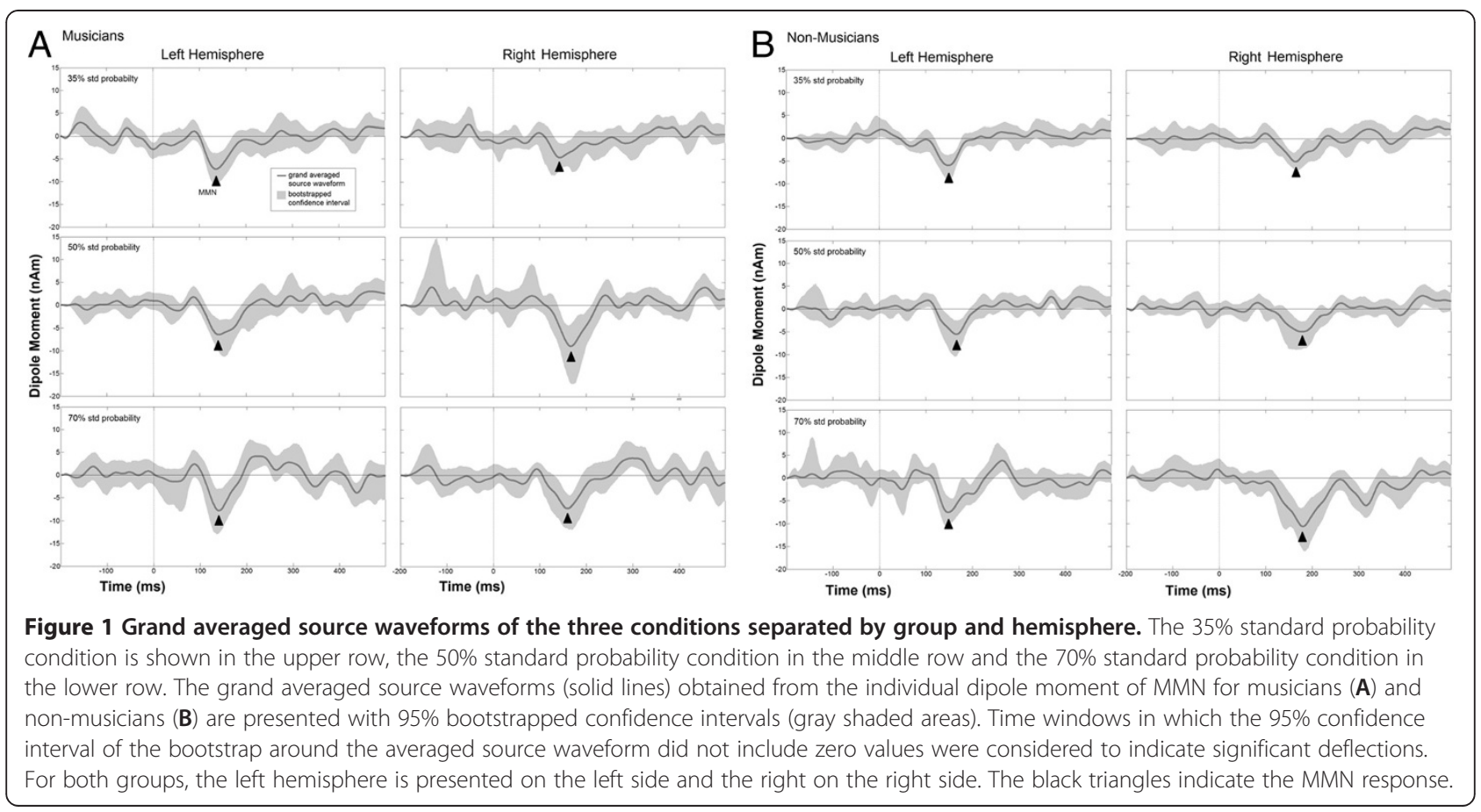


effect of standard probability in the right hemisphere [F $(2,16)=7.799, \mathrm{p}=.004]$ with a post-hoc Bonferroni pairwise comparison revealing a more pronounced MMN in the $70 \%$ compared to the $50 \%$ [mean difference $= \pm 6.071, \mathrm{p}=.022 \mathrm{]}$ and the $35 \%$ standard probability [mean difference $= \pm 7.705, \mathrm{p}=.003$ ] There was no significant effect in the left hemisphere. The results of the, paired-samples t-tests for non-musicians show a significant difference in amplitude between left and right hemisphere in the for the $70 \%$ standard probability condition $[\mathrm{t}(1,17)=2.679, \mathrm{p}=.016]$ and no effects in the other standard probability conditions. The interaction of hemisphere $\times$ standard probability in the non-musicians and musicians is depicted separately in Figure 2 and the corresponding waveforms in Figure 1 . All results of the pattern conditions are shown in Additional file 1.

The statistical analysis of the pitch oddball condition in a mixed model $2 \times 2$ ANOVA, with the between subject factor being group and the within subject factor being hemisphere revealed, for amplitude, as expected, no significant differences between groups or hemispheres.

\section{Behavioural data - questionnaire}

Immediately after the test, participants were asked two questions. The first question (Q1) asked if they had observed anything in particular about the auditory stimuli they had heard during the experiment. We expected that an affirmative answer would be followed by the report that they noticed regular tone patterns, which were occasionally interrupted by different tone patterns. The second question (Q2) asked them directly if they had noticed regular patterns and, if they had, in which run they had noticed them first. Three subjects missed to answer Q1: 2 musicians and 1 non-musician. Of the remaining 33 subjects, 11 out of 16 musicians and 7 out of 17 non-musicians reported detecting the presence of the tone patterns. Q2 was answered by 32 subjects (15 musicians and 17 non-musicians), 10 musicians and 6 non-musicians reported to have noticed the pattern structure. The difference in the answers (coded as correct and incorrect) between the groups in both questions, as revealed by an $x^{2}$-test, was not statistically significant, but there was a trend in the difference in Q2 in favor of the musicians $\left[x^{2}(1)=3.137, p=.077\right]$.

\section{Discussion}

In this study, we have shown how the statistical salience of sound patterns in an acoustic environment and longterm musical expertise affect the auditory encoding of such patterns. Musicians and non-musicians passively listened to three runs of tone pattern streams that consisted of different tone patterns. Within each run, one of the patterns had the highest probability of occurring, thus making it the standard pattern. The probability of this most frequent standard pattern differed between runs (salient condition 70\%, less salient condition $50 \%$ and least salient condition $35 \%$ standard probability). We observed a significant MMN response in all three conditions of acoustic salience, including the least salient condition (which featured a standard probability of $35 \%$ ), in both groups. The MMN amplitude of the non-musicians was influenced by standard probability and was more pronounced in the salient (70\% standard) condition than in the less and least salient (50\% and 35\% standard) conditions, especially in the right hemisphere. The musicians' MMN amplitudes, however, were not influenced by standard probability, leading to the

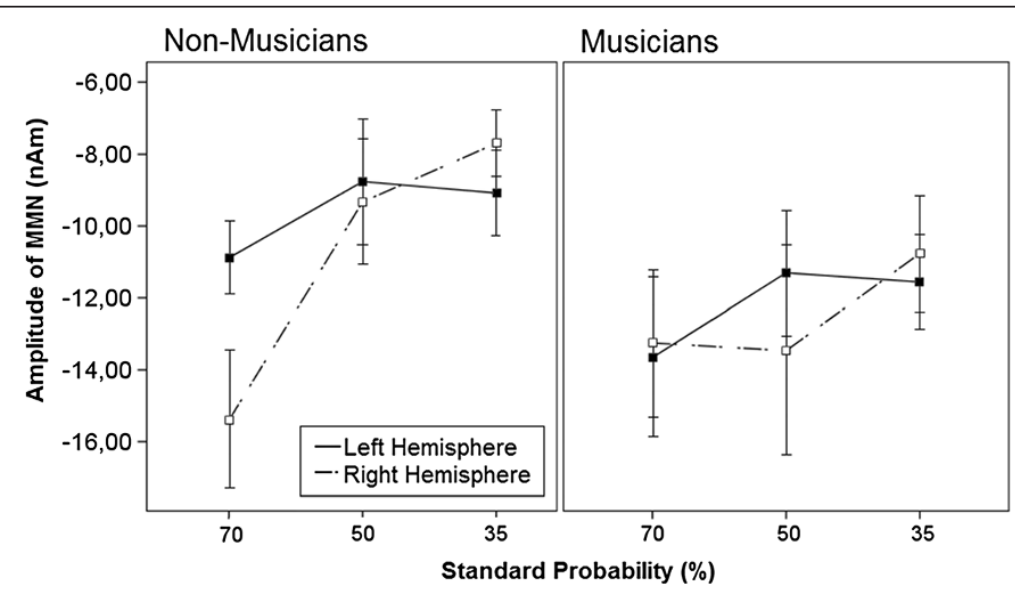

Figure 2 Interaction effect of hemisphere $\times$ standard probability in the two groups with corresponding standard error bars. The nonmusicians are presented at the left panel and the musicians at the right panel. The MMN amplitude of the right hemisphere in the non-musicians is significantly larger in the $70 \%$ condition than in the $50 \%$ and $35 \%$ conditions, whilst the amplitude of the left hemisphere remains stable. In the non-musicians, the MMN amplitude of the right hemisphere is significantly larger than in the left hemisphere in the $70 \%$ condition. There are no significant differences between conditions in the group of musicians. 
interpretation that the lateralized dependency of the MMN amplitude on salience was modulated by the factor long term musical training.

\section{MMN in an acoustic environment with a low level of salience}

In MMN study designs, the standard probability is very often set to an average of $70-90 \%$. For simple feature MMN, approaches such as the multi-features designs [46-49] have shown in an impressive way that MMN can be elicited with $50 \%$ standards or even without an actual standard [50]. However, we are not aware of studies that investigated the lower threshold of the standard probability in higher-order pattern processing. In the present study we observed an MMN response to tone pattern violations with a standard probability of only $35 \%$ in both musicians and non-musicians. As in the above mentioned multi-feature paradigms also in the present study a certain aspect of the standard representation has been reinforced on a local-regularity-level (single tone). The global-regularity level (pattern) on the other hand which had to be encoded first to understand the violations to the patterns the standard probability was in the lowest condition only $35 \%$. To our knowledge, this is the first demonstration that tone patterns can be encoded as the standard even at such a low level of salience.

This finding is in line with previous research, which has indicated that expectations can be formed on the basis of global characteristics of the auditory environment, such as the present tone patterns, and not only on the basis of local regularities [10-13]. It, furthermore, shows that the comparator mechanism underlying the mismatch negativity of the auditory system is far more sophisticated and sensitive to regularities in the auditory environment, even in the presence of high levels of statistical noise (deviants), than was assumed until now. In order to detect regularity violation, the regularity has first to be established, a process also known as standard formation [51]. The results of the present study suggest that the ability of our auditory system to form a standard representation in more complex designs, such as tone patterns, needs a surprisingly low signal (standards) among the noise (deviants). While other studies $[12,13,52]$ have used standard probabilities of $50 \%$, lower probability rates have not been used. Since, in our paradigm, all conditions yielded significant MMN deflections, further research is necessary to detect the lower boundary of standard probability that allows pattern detection to take place. On the basis of the present results, this lower threshold should be even less than $35 \%$. It would also be interesting to investigate the percentage of standard probability at which deviant detection enters a conscious stage and how it is influenced by musical expertise. This question could not be addressed within the current design but the behavioural data we obtained suggest that this may indeed be influenced by musical training. The focus of attention may also play a role [53,54].

\section{Hemispheric lateralization of pattern processing}

In line with previous studies which used simple feature oddball paradigms [18-20], we found that pattern MMN amplitude varied with standard probability at least in the non-musicians. One possible reason why Sculthorpe and Campbell [17] did not observe this relationship could be ceiling effects due to the different standard probabilities they used. The probabilities they used were much higher than in the present study and the deviants of each of that probabilities were, therefore, able to elicit pronounced MMN responses. In other words, increasing the standard probability over a certain level may not enhance the MMN amplitude any further. By using sufficiently different salience levels we were able to modulate MMN amplitude. The relationship between standard probability and MMN amplitude has been interpreted as evidence of a stronger memory trace due to easier formation of the standard. In a recent study Bendixen \& Schröger (2008) showed that the relationship of standard probability and MMN amplitude (or inverse relationship of deviant probability and MMN amplitude) is not observed for abstract regularities [55]. The correlation found in single-feature MMN was attributed by the authors to a contamination of the response by refractoriness. Nevertheless this contamination problem is not part of the design of the present study. This indicates that the explanation that MMN probability effects are due to N1 refractoriness as suggested by Bendixen \& Schröger (2008) and reasoned by Sculthorpe and Campbell (2011) may be too simple. Probabilities of abstract patterns modulate MMN amplitude in a way that seems independent of refractoriness.

We were, furthermore, interested in the role of the right and left auditory cortices in tone pattern processing. As can be seen in Figure 1, the standard probability mainly affects the $\mathrm{MMN}$ response in the right hemisphere of non-musicians, while responses in the left hemisphere of non-musicians, and in both hemispheres of musicians were not significantly affected by standard probability across the different conditions. Previous work has shown that acoustic stimuli, such as music and tones, are predominantly processed in the right auditory cortices [1,3,56,57]. On closer examination, however, this lateralization seems to be dependent on the particular stimulus parameters involved. The processing of tone patterns, for example, was lateralized towards the left hemisphere in previous MMN studies $[12,13,41]$. Lateralization of tone processing is also modulated by the rhythmic and metric structure of tone sequences, familiarity, top-down expectations and musical expertise [40,56,58-61]. Processing can even shift from one 
hemisphere to the other when arbitrary stimuli become meaningful in the course of a short-term training procedure [62]. In the present study, tone patterns were used as stimulus material and, whereas the results did not show an overall lateralization of processing, we found a modulation of the MMN amplitude by the standard probability only in the right hemisphere of non-musicians, while the left hemisphere was not significantly affected by standard probability. This may imply that the processing of tone patterns in a salient environment is right lateralized, but as the level of salience (deviant probability) decreases and the regularities are more difficult to encode, additional processing in the left hemisphere is required.

\section{Influence of musical expertise}

A large body of literature shows that musical experience, in both the long and short term, modulates the processing of auditory material, especially for stimuli such as complex tone patterns $[12,13,40,63,64]$. In the present study, auditory processing of tone patterns in the musicians was not influenced by the standard probability, but this influence was apparent in the case of the nonmusicians. The non-musicians' right hemispheres were more strongly influenced than the left hemispheres, especially in the $70 \%$ standard probability condition. This implies that the auditory processing of musicians is not dependent on the level of salience as used in our study, albeit it could be possible that with even lower standard probabilities an influence on the MMN could be seen also in musicians. Previous research has shown that the processing of more complex material, such as tone patterns, as opposed to the classic oddball paradigm stimuli, is facilitated by musical expertise $[12,59,61,65]$, possibly because formal musical training would direct musicians toward more analytical processing of acoustic stimuli. Although the high standard probability stimuli used in the current study are not as simple as the classic oddball material, we consider them to be simpler than the lower standard probability stimuli (essentially, the lower the standard probability, the higher the complexity) and, as such, the results of the current study fit well with previous research findings regarding musicians' greater facility for processing of complex auditory stimuli. These superior auditory processing abilities may explain the absence of an effect of standard probability upon the musicians. This demonstration that musicians are less affected by the variability of the signal in the detection of acoustic regularities reflects their expertise in the auditory domain.

\section{Conclusion}

The results indicate that the MMN amplitude in the right hemisphere of non-musicians in response to deviants in tone patterns is influenced by the probability of the occurrence of the standard pattern, with the effect being greater for more salient acoustic stimuli. The amplitude of the MMN response in the left hemisphere of nonmusicians is more stable. The MMN response in musicians, on the other hand, did not seem to be influenced by the level of salience. This implies that violation detection processing in non-musicians (ie. comparative non-experts in auditory processing) is dependent on the salience of the acoustic environment: in acoustic environments with a low level of salience, detection of change is more challenging for the auditory networks than in a more salient acoustic environment.

\section{Methods}

Subjects

21 musicians and 22 non-musicians participated in the experiment. Seven subjects (3 musicians, 4 non-musicians), were excluded from the final analysis due to insufficient MEG recording quality, excessive head movements, or insufficient quality of the model fit of their recorded data (exclusion criteria: the dipoles explained less than $85 \%$ of the magnetic field variance) resulting in a total number of 36 subjects (18 musicians, mean age 24.61 (SD 2.81), 7 males; 18 non-musicians, mean age 25.5 (SD 2.85), 6 males). Musicians were students at the Music Conservatory in Münster or professionals or had received extensive musical training since childhood (minimum ten years) and were still actively playing (average practice time of 18.11 hours per week) as evaluated by a questionnaire; none of them had absolute pitch (self-report). Non-musicians were classified by not having received any musical training apart from basic compulsory music classes in school. All subjects were right-handed as assessed by the Edinburgh Handedness Inventory [66], had normal hearing as assessed by clinical audiometry, and provided written consent prior to their participation in the study. The study protocol was approved by the ethics committee of the Medical Faculty of the University of Münster and the study was conducted according to the Declaration of Helsinki.

\section{Stimuli and procedure}

The tones were generated in $44100 \mathrm{~Hz}$ stereo and $32 \mathrm{bit}$, the frequencies used were $W=500 \mathrm{~Hz}, X=594 \mathrm{~Hz}$, $\mathrm{Y}=705 \mathrm{~Hz}$ and $\mathrm{Z}=838 \mathrm{~Hz}$, which equate to ascending tones with a pitch difference of three semitones. The duration of each tone was $200 \mathrm{~ms}$ including $10 \mathrm{~ms}$ rise and decay time, and the interstimulus interval was set to $400 \mathrm{~ms}$. Each trial consisted of three tones forming one tone pattern. Tone patterns always started with tone W. Half of the deviants were pure pattern deviants, in the sense that they contained no tones of a different frequency than the tones of the standard pattern, and the other half were combined frequency and pattern deviants that contained one tone with a different frequency 
than the tones of the standard pattern. Examples for both types of deviants are depicted in Figure 3. Three different tone patterns were used as standard patterns: WXY (with corresponding deviant patterns W $\underline{Y} Y, W \underline{Z} Y$, WXZ and WXX), WYZ (corresponding deviant trials WXXZ, WZZZ, WYX $\underline{X}$ and WYY ) and WZX (corresponding

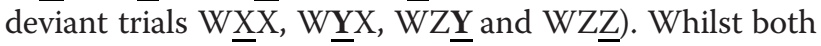
pattern (violating only the pattern but not the pitch of the standard stimuli) and combined pattern and frequency (violating the pattern as well as the pitch of the standard stimuli) deviants were analysed, we focused our interpretation on the results for the pure pattern deviants because we were mainly interested in the higher order regularities. The combined pattern and frequency deviants were only included into the stimulus material to increase the number of different deviant patterns rendering any of the deviant patterns less probable than the standard pattern but could not be analyses due to refractoriness confounds introduced by the additional tone frequency which occurs more rarely than any of the other tones in the sequence. The probabilities of the 4 different deviant patterns were the following: 7 to $8 \%$ in the $70 \%$ standard pattern condition, 10 to $15 \%$ in the $50 \%$ standard pattern condition and 15 to $20 \%$ in the $35 \%$ standard pattern condition (with decreasing stan- dard probability the deviant probability was increasing, accordingly). The mismatch between standard and deviant pattern in both deviant types could either be at the second or the third position of the pattern (as indicated by underscore, see above), and each possibility was presented in half of the deviant trials. The presentation of the stimulus material (the 3 different standard pattern conditions and the oddball condition) was divided into 4 runs of approximately 12 minutes each. One out of the three standard probabilities $(70 \%=$ salient, $50 \%=$ less salient and $35 \%=$ least salient) was presented in each of the 2nd to 4th) run, and counterbalanced across standard tone pattern and run order amongst the subjects. The tone patterns were presented as a continuous sound stream with no perceivable gaps between consecutive tone patterns. In total, 400 trials were presented for each run of the each of three pattern conditions.

We used a classic frequency oddball paradigm $(500 \mathrm{~Hz}$ and $530 \mathrm{~Hz}$ tones, one semitone difference) as a control condition in which a reliable mismatch response was expected in all subjects. This condition was presented before the three pattern conditions in run 1 . The tones were presented as continuous stream with tone duration of $200 \mathrm{~ms}$ including $10 \mathrm{~ms}$ rise and decay and an ISI of $500 \mathrm{~ms}$. In total, 995 tones were presented. The
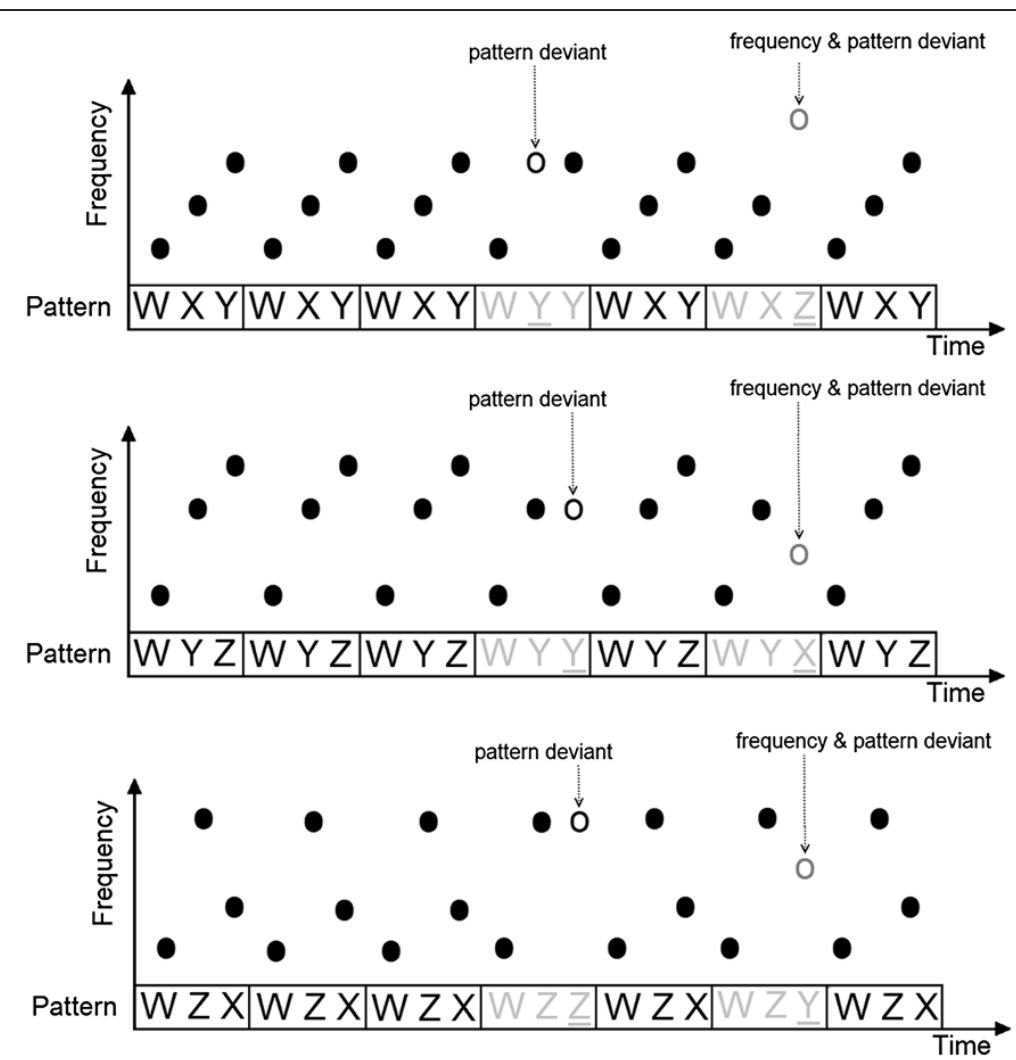

Figure 3 Outline of the three standards of the pattern condition (standard, pattern deviant, and frequency and pattern deviant). The patterns were embedded in a continuous sound stream with standard pattern probability set to $35 \%, 50 \%$ and $70 \%$, respectively. 
probability of deviant occurrence was set at 0.15 and at least 3 standards preceded each deviant

Participants passively listened to all conditions while they were attending to a silent movie (Disney's "Peter Pan"). After each run, they had to answer four to six questions about the content of the movie to ensure that they paid attention. After the last run, participants were debriefed and asked if they noticed anything in particular about the acoustic stimuli. If they did not they were specifically asked if they noticed a pattern in the acoustic stimuli and, if they did, during which run they first noticed it. The overall duration of the experiment was approximately 1.5 hours and the total time of the MEG recordings was approximately 48 minutes.

\section{MEG recordings}

Magnetic fields were recorded with a 275 channel whole-head system (OMEGA, CTF Systems Inc, Port Coquitlam, Canada) in an acoustically and magnetically shielded room. MEG data were acquired continuously during presentation blocks with a sampling rate of $600 \mathrm{~Hz}$. The subjects passively listened to the four blocks (one run of pitch oddball condition, three runs of pattern conditions) with short breaks in between, during which they could relax and were asked questions about the content of the movie by the investigator. Participants were seated upright and their head position was comfortably stabilized with pads inside the dewar. Stimuli were delivered via air conduction through plastic tubes at $60 \mathrm{~dB}$ above the individual hearing threshold, which was determined for each ear at the beginning of each MEG session for the different stimuli with an accuracy of $5 \mathrm{~dB}$. The subject's alertness, well-being and compliance were verified by video monitoring. The subjects were instructed to minimize swallowing and blinking.

\section{Data analysis}

The continuous data were separated into epochs of 600 $\mathrm{ms}$, starting $100 \mathrm{~ms}$ before and ending $500 \mathrm{~ms}$ after the tone onset of the deviant tone of a deviant pattern, or the corresponding standard tone of a standard pattern (the standard tone at the same position as the deviant tone analyzed) in all 3 pattern conditions (35\%, 50\%, $70 \%)$. In the pitch oddball condition analogous epochs of $600 \mathrm{~ms}$ were extracted from all deviants and every second standard before a deviant. Epochs containing signal amplitudes larger than $2.5 \mathrm{pT}$ were considered artifacts and were excluded from averaging. Baseline correction was based on the $100 \mathrm{~ms}$ baseline before the tone onset of each epoch. Standards and deviants were averaged separately and digitally filtered (high pass filter of $1 \mathrm{~Hz}$ and a low pass filter of $30 \mathrm{~Hz}$ ). Averaged responses to standards were subtracted from averaged responses to deviants in order to acquire the difference response containing the $\mathrm{MMN}$ in all conditions.

In the analysis of the data, two equivalent current dipoles (ECD), one in each hemisphere, were used to model the MMN field, a technique justified by the dipolar distribution of the MMN [67].

The ECDs were fitted simultaneously in a spherical volume conductor to each individual's peak of MMN (restricted to the predefined MMN window of 120 to $250 \mathrm{~ms}$ ) in the averaged difference response. Source waveforms for each of the participants in each of the conditions were derived from the MEG data using the technique of signal space projection [68], thereby reducing the data to one source waveform for each hemisphere. MMN sources are assumed to remain relatively stable across similar stimulations and the source space projection method is robust to slight displacements of sources. The fit from the model with the best signal-tonoise-ratio was used for all conditions within one subject. All dipolar sources included in the analysis explained at least $85 \%$ of the magnetic field variance with a mean goodness of fit of $90.7 \%$ and no significant difference between the groups was found with an independent sample t-test $([\mathrm{T}(1,34)=.382, \mathrm{p}=.705]$, mean goodness of fit for $\mathrm{NM}=90.87 \%, \mathrm{M}=90,53 \%)$.

\section{Additional file}

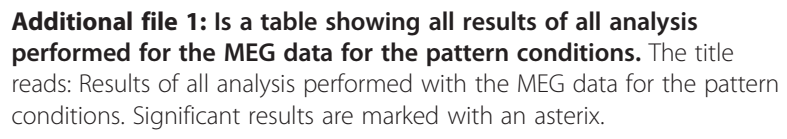

Competing interests

The authors declare that they have no competing interests.

\section{Authors' contributions}

AK, EP, SCH and CP designed the experiment and interpreted the results; AK carried out data acquisition and analysis and drafted the manuscript; EP, SCH and $C P$ revised the manuscript. All authors read and approved the final manuscript.

\section{Acknowledgements}

We would like to thank our test subjects for their cooperation, our technicians for supporting the data acquisition, A. Wollbrink for technical help and Ross Parfitt for his helpful comments. We acknowledge support by Deutsche Forschungsgemeinschaft [PA392/12-2 and HE6067-1/1] and Open Access Publication Fund of University of Muenster.

\section{Author details}

${ }^{1}$ Institute for Biomagnetism and Biosignalanalysis, University of Münster, Münster, Germany. ${ }^{2}$ Montreal Neurological Institute, McGill University, Montreal, Quebec, Canada. ${ }^{3}$ German Center for Neurodegenerative Diseases (DZNE), Bonn, Germany.

Received: 28 November 2012 Accepted: 20 April 2013 Published: 24 April 2013 


\section{References}

1. Kujala T, Tervaniemi M, Schröger E: The mismatch negativity in cognitive and clinical neuroscience: theoretical and methodological considerations. Biol Psychol 2007, 74:1-19.

2. Näätänen R, Paavilainen P, Rinne T, Alho K: The mismatch negativity (MMN) in basic research of central auditory processing: a review. Clin Neurophysiol 2007, 118:2544-2590.

3. Picton TW, Alain C, Otten L, Ritter W, Achim A: Mismatch negativity: different water in the same river. Audiol Neurootol 2000, 5:111-139.

4. Baldeweg T, Richardson A, Watkins S, Foale C, Gruzelier JH: Impaired auditory frequency discrimination in dyslexia detected with mismatch evoked potentials. Ann Neurol 1999, 45:495-503.

5. Jessen F, Fries T, Kucharski C, Nishimura T, Hoenig K, Maier W, Falkai P, Heun $R$ : Amplitude reduction of the mismatch negativity in first-degree relatives of patients with schizophrenia. Neurosci Lett 2001, 309:185-188.

6. Csépe V, Karmos G, Molnár M: Evoked potential correlates of stimulus deviance during wakefulness and sleep in cat-animal model of mismatch negativity. Electroencephalogr Clin Neurophysiol 1987, 66:571-578.

7. Javitt DC, Schroeder CE, Steinschneider M, Arezzo JC, Vaughan HG Demonstration of mismatch negativity in the monkey. Electroencephalogr Clin Neurophysiol 1992, 83:87-90.

8. Nelken I, Ulanovsky N: Mismatch negativity and stimulus-specific adaptation in animal models. J Psychophysiology 2007, 21:214-223.

9. Sams M, Hari R, Rif J, Knuutila J: The human auditory sensory memory trace persists about $10 \mathrm{sec}$ : neuromagnetic evidence. J Cogn Neurosci 1993, 5:363-370

10. Sussman E, Sheridan K, Kreuzer J, Winkler I: Representation of the standard: stimulus context effects on the process generating the mismatch negativity component of event-related brain potentials. Psychophysiology 2003, 40:465-471.

11. Sussman E, Winkler I: Dynamic sensory updating in the auditory system. Brain Res Cogn Brain Res 2001, 12:431-439.

12. Herholz SC, Lappe C, Pantev C: Looking for a pattern: an MEG study on the abstract mismatch negativity in musicians and nonmusicians. BMC Neurosci 2009, 10:42.

13. Kuchenbuch A, Paraskevopoulos E, Herholz SC, Pantev C: Electromagnetic Correlates of Musical Expertise in Processing of Tone Patterns. PLoS One 2012, 7:e30171.

14. Ulanovsky N, Las L, Farkas D, Nelken I: Multiple time scales of adaptation in auditory cortex neurons. J Neurosci 2004, 24:10440-10453.

15. Von der Behrens W, Bäuerle P, Kössl M, Gaese BH: Correlating stimulus-specific adaptation of cortical neurons and local field potentials in the awake rat. J Neurosci 2009, 29:13837-13849.

16. Farley BJ, Quirk MC, Doherty JJ, Christian EP: Stimulus-specific adaptation in auditory cortex is an NMDA-independent process distinct from the sensory novelty encoded by the mismatch negativity. J Neuroscience 2010, 30:16475-16484.

17. Sculthorpe LD, Campbell KB: Evidence that the mismatch negativity to pattern violations does not vary with deviant probability. Clin Neurophysiol 2011, 122:2236-2245.

18. Sato Y, Yabe H, Hiruma T, Sutoh T, Shinozaki N, Nashida T, Kaneko S: The effect of deviant stimulus probability on the human mismatch process. Neuroreport 2000, 11:3703-3708.

19. Sabri M, Campbell KB: Effects of sequential and temporal probabilty of deviant occurence on mismatch negativity. Cogn Brain Res 2001, 12:171-180.

20. Sonnadara RR, Alain C, Trainor LJ: Effects of spatial separation and stimulus probability on the event-related potentials elicited by occasional changes in sound location. Brain Res 2006, 1071:175-185.

21. Javitt DC, Grochowski S, Shelley a M, Ritter W: Impaired mismatch negativity (MMN) generation in schizophrenia as a function of stimulus deviance, probability, and interstimulus/interdeviant interval. Electroencephalogr Clin Neurophysiol 1998, 108:143-153.

22. Pincze Z, Lakatos P, Rajkai C, Ulbert I, Karmos G: Effect of deviant probability and interstimulus/interdeviant interval on the auditory $\mathrm{N} 1$ and mismatch negativity in the cat auditory cortex. Brain Res Cogn Brain Res 2002, 13:249-253.

23. Campitelli G, Gobet F, Head K, Buckley M, Parker A: Brain localization of memory chunks in chessplayers. Int I Neurosci 2007, 117:1641-1659.

24. Campitelli G, Gobet F, Parker A: Structure and stimulus familiarity: a study of memory in chess-players with functional magnetic resonance imaging. Span J Psychol 2005, 8:238-245.
25. May A: Experience-dependent structural plasticity in the adult human brain. Trends Cogn Sci 2011, 15:475-482.

26. Woollett K, Maguire E: Acquiring "the Knowledge" of London's layout drives structural brain changes. Current biology: CB 2011, 21:2109-2114.

27. Maguire EA, Woollett $K$, Spiers HJ: London taxi drivers and bus drivers: a structural MRI and neuropsychological analysis. Hippocampus 2006, 16:1091-1101

28. Hyde KL, Lerch J, Norton A, Forgeard M, Winner E, Evans AC, Schlaug G: Musical training shapes structural brain development. J Neurosci 2009, 29:3019-3025

29. Jäncke L: Music drives brain plasticity. F1000 biology reports 2009, 1:1-6.

30. Jäncke L: The plastic human brain. Restor Neurol Neurosci 2009, 27:521-538.

31. Münte TF, Altenmüller $E$, Jäncke $L$ : The musician's brain as a model of neuroplasticity. Nat Rev Neurosci 2002, 3:473-478.

32. Pantev C, Lappe C, Herholz SC, Trainor LJ: Auditory-somatosensory integration and cortical plasticity in musical training. Ann N Y Acad SCi 2009, 1169:143-150.

33. Zatorre RJ: Music, the food of neuroscience ? Nature 2005, 434:312-315.

34. Zatorre RJ, Belin P, Penhune VB: Structure and function of auditory cortex: music and speech. Trends Cogn Sci 2002, 6:37-46.

35. Tervaniemi M, Rytkönen M, Schröger E, IImoniemi RJ, Näätänen R: Superior formation of cortical memory traces for melodic patterns in musicians. Learning \& Memory (Cold Spring Harbor, N.Y.) 2001, 8:295-300.

36. Münte TF, Nager W, Beiss T, Schroeder C, Altenmüller E: Specialisation of the specialized: electrophysiological investigations in professional musicians. Ann N Y Acad Sci 2003, 999:131-139.

37. Nager W, Kohlmetz C, Altenmüller E, Rodriquez-Fornells A, Münte TF: The fate of sounds in conductors' brains: an ERP study. Brain Res Cogn Brain Res 2003, 17:83-93.

38. Wan $\mathrm{CY}$, Schlaug G: Music making as a tool for promoting brain plasticity across the life span. Neuroscientist 2010, 16:566-577.

39. Besson M, Faïta F, Requin J: Brain waves associated with musical incongruities differ for musicians and non-musicians. Neurosci Lett 1994, 168:101-105.

40. Boh B, Herholz SC, Lappe C, Pantev C: Processing of complex auditory patterns in musicians and nonmusicians. PLoS One 2011, 6:e21458.

41. Herholz SC, Boh B, Pantev C: Musical training modulates encoding of higher-order regularities in auditory cortex. Eur J Neurosci 2011, 34:524-529.

42. Darvas F, Rautiainen M, Baillet S, Ossadtchi A, Mosher JC, Leahy RM: Investigations of dipole localisation accuracy in MEG using the bootstrap. Neuroimage 2005, 25:355-368.

43. Efron B: Bootstrap methods: another look at the jackknife. Ann Stat 1979, 7:1-26.

44. Di Nocera F, Ferlazzo F: Resampling approach to statistical inference: bootstrapping from event-related potentials data. Behav Res Methods Instrum Comput 2000, 32:111-119.

45. Johnson RW: An Introduction to the Bootstrap. Teach Stat 2001, 23:49-54.

46. Pakarinen S, Takegata R, Rinne T, Huotilainen M, Näätänen R: Measurement of extensive auditory discrimination profiles using the mismatch negativity (MMN) of the auditory event-related potential (ERP). Clin Neurophysiol 2007, 118:177-185.

47. Näätänen R, Pakarinen S, Rinne T, Takegata R: The mismatch negativity (MMN): towards the optimal paradigm. Clin Neurophysiol 2004, 115:140-144.

48. Vuust P, Brattico E, Glerean E, Seppänen M, Pakarinen S, Tervaniemi M, Näätänen R: New fast mismatch negativity paradigm for determining the neural prerequisites for musical ability. Cortex 2011, 47:1091-1098.

49. Pakarinen S, Lovio R, Huotilainen M, Alku P, Näätänen R, Kujala T: Fast multi-feature paradigm for recording several mismatch negativities (MMNs) to phonetic and acoustic changes in speech sounds. Biol Psychol 2009, 82:219-226

50. Pakarinen $S$, Huotilainen $M$, Näätänen $R$ : The mismatch negativity (MMN) with no standard stimulus. Clin Neurophysiol 2010, 121:1043-1050.

51. Sussman E: A new view on the MMN and attention debate. J Psychophysiology 2007, 21:164-175.

52. Fujioka T, Trainor LJ, Ross B, Kakigi R, Pantev C: Automatic encoding of polyphonic melodies in musicians and nonmusicians. J Cogn Neurosci 2005, 17:1578-1592.

53. Sussman E, Winkler I, Wang W: MMN and attention: competition for deviance detection. Psychophysiology 2003, 40:430-435. 
54. Sussman E, Horváth J, Winkler I, Orr M: The role of attention in the formation of auditory streams. Percept Psychophys 2007, 69:136-152.

55. Bendixen A, Schröger E: Memory trace formation for abstract auditory features and its consequences in different attentional contexts. Biol Psychol 2008, 78:231-241

56. Tervaniemi M, Hugdahl K: Lateralization of auditory-cortex functions. Brain Res Rev 2003, 43:231-246.

57. Okamoto H, Stracke H, Bermudez P, Pantev C: Sound processing hierarchy within human auditory cortex. J Cogn Neurosci 2011, 23:1855-1863.

58. Shahin AJ, Roberts LE, Pantev C, Aziz M, Picton TW: Enhanced anteriortemporal processing for complex tones in musicians. Clin Neurophysiol 2007, 118:209-220.

59. Vuust P, Pallesen K, Bailey CJ, Van Zuijen TL, Gjedde A, Roepstorff A, Østergaard L: To musicians, the message is in the meter pre-attentive neuronal responses to incongruent rhythm are left-lateralized in musicians. Neuroimage 2005, 24:560-564.

60. Lappe C, Herholz SC, Trainor L, Pantev C: Cortical plasticity induced by short-term unimodal and multimodal musical training. J Neurosci 2008, 28:9632-9639.

61. Fujioka T, Trainor $\sqcup$, Ross B, Kakigi R, Pantev C: Automatic encoding of polyphonic melodies in musicians and nonmusicians. J Cogn Neurosci 2005, 17:1578-1592. doi:10.1162/089892905774597263.

62. Kujala A, Huotilainen $M$, Uther M, Shtyrov $Y$, Monto S, Ilmoniemi RJ, Näätänen R: Plastic cortical changes induced by learning to communicate with non-speech sounds. Neuroreport 2003, 14:1683-1687.

63. Näätänen R: Mismatch negativity: clinical research and possible applications. Int J Psychophysiol 2003, 48:179-188.

64. Wang W, Staffaroni L, Reid E, Steinschneider M, Sussman E: Effects of musical training on sound pattern processing in high-school students. Int J Pediatr Otorhinolaryngol 2009, 73:751-755.

65. Herholz SC, Lappe C, Knief A, Pantev C: Neural basis of music imagery and the effect of musical expertise. Eur J Neurosci 2008, 28:2352-2360.

66. Oldfield RC: The assessment and analysos of handedness: the edinburgh inventory. Neuropsychologia 1971, 9:97-113.

67. Csépe V, Pantev C, Hoke M, Hampson S, Ross B: Evoked magnetic responses of the human auditory cortex to minor pitch changes: localization of the mismatch field. Electroencephalogr Clin Neurophysiol 1992, 84:538-548.

68. Tesche CD, Uusitalo MA, IImoniemi RJ, Huotilainen M, Kajola M, Salonen O: Signal-space projections of MEG data characterize both distributed and well-localized neuronal sources. Electroencephalogr Clin Neurophysiol 1995, 95:189-200.

doi:10.1186/1471-2202-14-51

Cite this article as: Kuchenbuch et al:: Effects of musical training and event probabilities on encoding of complex tone patterns. BMC Neuroscience 2013 14:51.

\section{Submit your next manuscript to BioMed Central and take full advantage of:}

- Convenient online submission

- Thorough peer review

- No space constraints or color figure charges

- Immediate publication on acceptance

- Inclusion in PubMed, CAS, Scopus and Google Scholar

- Research which is freely available for redistribution

Submit your manuscript at www.biomedcentral.com/submit
Ciomed Central 